\title{
Papel Mediador das Representações do Tabaco e do Suporte do Parceiro em Fumantes e Ex-Fumantes
}

\author{
Fernanda Afonso ${ }^{1}$ \\ M. Graça Pereira \\ Escola de Psicologia da Universidade do Minho, Braga, Portugal
}

\section{Resumo}

Este estudo avaliou as representações associadas ao tabaco como variável mediadora na relação entre o ajustamento de casal e o suporte do parceiro em fumantes e ex-fumantes, bem como o papel do suporte do parceiro como mediador na relação entre as representações do tabaco e variáveis sócio-cognitivas para deixar de fumar. Participaram no estudo 224 fumantes e 169 ex-fumantes. Os fumantes fumavam há, pelo menos, 3 anos e os ex-fumantes tinham deixado de fumar há mais de 3 meses. Os resultados mostraram, nos fumantes, que o suporte do parceiro apresentou um efeito mediador parcial na relação entre as representações do tabaco e as variáveis sócio-cognitivas (atitudes, controle comportamental percebido e planejamento do coping/ação). As representações emocionais do tabaco não tiveram um papel mediador na relação entre o ajustamento de casal e o suporte do parceiro, em fumantes e ex-fumantes. Os resultados apontam para a importância do suporte do parceiro na intervenção junto de fumantes com intenção para deixar de fumar.

Palavras-chaves: Representações do tabaco, suporte do parceiro, mediadores, fumantes, ex-fumantes.

\section{Mediator Role of Tobacco Representations and Partner Support in Smokers and Abstinents}

\begin{abstract}
This study evaluated the representations associated with tobacco as a mediator between couple's adjustment and partner support in smokers and abstinents, as well as the role of the partner's support as a mediator in the relationship between the representations associated with tobacco and sociocognitive variables to quit smoking. 224 smokers and 169 abstinent participated in the study. Smokers smoked at least for 3 years and abstinents were smoke free for more than 3 months. Results revealed that partner support had a mediator effect on the relationship between the representations associated with tobacco and sociocognitive variables (attitudes, perceived behavioral control and planning of coping/action). The emotional representations of tobacco were not a mediator role in the relationship between the couple adjustment and partner support on smokers and abstinents. Results indicated the importance of including partner support during smoking cessation interventions among smokers with intention to quit smoking.
\end{abstract}

Keywords: Tobacco representation, partner support, mediators, smokers, abstinents.

Endereço para correspondência: Departamento de Psicologia Aplicada, Escola de Psicologia, Universidade do Minho, Campus de Gualtar, Braga, Portugal 4710-057. E-mail: fernandafonso@gmail.com e gracep@psi. uminho.pt 


\section{Papel Mediador de las Representaciones del Tabaco y del Apoyo del Cónyuge en Fumadores y Abstinentes}

\section{Resumen}

Este estudio evaluó las representaciones asociadas al tabaco como una variable mediadora en la relación entre el ajuste familiar y el apoyo del cónyuge en los fumadores y los ex-fumadores, y el papel de apoyo del cónyuge como mediador en la relación entre las representaciones asociadas al tabaco y las variables socio-cognitivas para dejar de fumar. 224 fumadores y 169 ex-fumadores participaron en el estudio. La duración del consumo en el grupo de fumadores fue de al menos 3 años y la duración de la abstinencia superior a 3 meses. Los resultados mostraron, en los fumadores, el apoyo de la pareja tenía un efecto mediador parcial en la relación entre las representaciones asociadas al tabaco y las variables socio-cognitivas (actitudes, control comportamental percibido e planificación del coping/ acción). Las representaciones emocionales no tenían un papel mediador en la relación entre el ajuste familiar y el apoyo del cónyuge en los fumadores y los ex-fumantes. Los resultados señalan la importancia del apoyo del cónyuge en la intervención con los fumadores que deseen dejar de fumar.

Palabras clave: Representaciones del tabaco, apoyo del cónyuge, mediadores, fumadores, ex-fumadores.

Fumar é um grave problema com consequências graves para a saúde (Becoña, 2003). O tabaco é percebido pela maioria dos fumantes como um comportamento normal e inofensivo e com consequências, apenas, a longo prazo (Martinet \& Bohadana, 2003). Em Portugal, no período entre 2005 e 2006 , cerca de $19,6 \%$ da população de Portugal Continental eram fumantes, e 15,4\% eram ex-fumantes (Direção Geral da Saúde [DGS], 2009). Assim, deixar de fumar continua a ser uma tarefa complexa e representa o passo mais importante dos fumantes para viverem mais tempo e melhorarem a sua qualidade de vida (Instituto Nacional de Cardiologia Preventiva [INCP], 2002; Lopez, Mathers, Ezzati, Jamison, \& Murray, 2006). Ao nível das representações associadas ao tabaco, muitos fumantes não reconhecem que fumar é um problema de saúde, que cria dependência e vulnerabilidade física possuindo assim representações desajustadas acerca do seu consumo de tabaco (Martinet \& Bohadana, 2003; Trigo, 2005) continuando a fumar (Afonso, 2008). As representações do tabaco podem ser agrupadas em representações cognitivas do tabaco, representações emocionais do tabaco e compreensão. As representações cognitivas indicam o quanto ameaçador as consequências do tabaco, a duração do hábito de fumar, o controle que o fumador tem sobre o comportamento de fumar, a eficácia do tratamento para deixar de fumar e como o individuo identifica os sintomas relacionados com o facto de fumar. As representações emocionais contemplam a preocupação associada ao fumar e o quanto o fumante se sente afetado emocionalmente pelo seu consumo de tabaco. A compreensão contempla o quanto o individuo compreende o seu comportamento de fumar. Quanto mais ameaçadoras as representações cognitivas, emocionais e menor a compreensão, mais o fumante considera que o tabaco é uma ameaça para a sua saúde (Broadbent, Petrie, Main, \& Weinman, 2006). Assim o individuo pode continuar a fumar mesmo estando consciente dos malefícios para a saúde (Meneses, Ribeiro, \& Silva, 2002). A literatura mostra que o suporte do parceiro é uma variável importante na mudança do comportamento tabágico fazendo parte de uma estratégia mais eficaz de ajudar os fumantes a deixar o seu hábito (Park, Tudiver, Schultz, \& Campbell, 2004; Rüge et al., 2008). Este apoio do parceiro refere-se ao tipo de apoio que os esposos, ou cônjuges, proporcionam ao fumante para deixar de fumar e tem-se revelado importante no sucesso das intervenções para a desabituação tabágica (Shoham, Rohrbaugh, Trost, \& Muramoto, 2006). Um estudo realizado por Thomas et al. (2009) avaliou 84 fumantes que foram atendidos 
em consultas de cessação tabágica, acompanhados dos respetivos companheiros. Foi verificado que a maioria dos parceiros dos fumantes tinham vontade de ajudar e demonstravam interesse em saber como o fazer. Assim, o apoio do parceiro pode ser considerado um dos melhores preditores para a abstinência tabágica (Lawhon, Humfleet, Hall, Reus, \& Muñoz, 2009; Nollen et al., 2005), pode influenciar o fumante a deixar de fumar e a manter-se abstinente (Homish \& Kenneth, 2005; Rüge et al., 2008), e é considerado preditivo do sucesso da desabituação tabágica. Este suporte do parceiro pode ser positivo (parceiro elogiar e apoiar de forma positiva o fumante) ou negativo (quando o parceiro tem uma atitude de vigilância constante e insistência para que o fumador pare de fumar; Cohen \& Lichtenstein, 1990; Copotelli \& Orleans, 1985; Mermelstein, Lichtenstein, \& McIntyre, 1983; Roski, Schmid \& Landom, 1996). Deste modo, a literatura realça a importância do suporte do parceiro na mudança do comportamento tabágico (Afonso, 2008; Mermelstein et al., 1983). Ao nível do ajustamento de casal Rohrbaugh, Shoham e Dempsey (2009) e Rohrbaugh et al. (2001) também referem a importância do parceiro no comportamento de fumar. Estudos enfatizaram a comunicação e a regulação de emoções entre o casal, no processo de deixar de fumar, como forma de aproximação ou distanciamento (Wenner, Rohrbaugh, Shoham, \& Trost, 2004). Assim, se um dos membros do casal fumar, e o outro não, o comportamento de fumar pode aproximar o casal ou afastá-lo. Os autores referem que uma intervenção focada no apoio do parceiro é mais eficaz quando comparado com as intervenções que se baseiam apenas no suporte social geral (Rohrbaugh et al., 2001). No que respeita às variáveis sócio-cognitivas, a intenção para deixar de fumar revela-se como um aspeto chave na Teoria do Comportamento Planejado (TCP), bem como um preditor, e mesmo um mediador, importantes do comportamento para deixar de fumar (Ajzen, 1991; Ajzen \& Madden, 1986; Fishbein \& Ajzen, 1975). A TCP tem sido aplicada a diversos comportamentos, relacionados com a saúde, entre os quais, a cessação tabágica (Moan \& Rise, 2005; Rise, Kovac, Kraft, \& Moan, 2008) e o consumo de tabaco (Hill, Boudreau, Amyot, De'ry, \& Godin,
1997). A intenção resulta da combinação das atitudes, normas subjetivas e controle percebido. Atitudes mais fortes, por oposição às fracas, têm uma maior probabilidade de predizer as intenções (Fila \& Smith, 2006). Pelo contrário, os indivíduos que creem não ter recursos para realizar um comportamento têm menor probabilidade de formar intenções para a sua realização, mesmo mantendo atitudes favoráveis e acreditarem que os outros aprovam a sua realização. Assim, se um fumante quer deixar de fumar (atitude), a família poderá apoiar esta decisão disponibilizando-se para o ajudar (norma subjetiva). No entanto, se o fumante apresentar uma baixa perceção da sua competência para deixar de fumar (perceção de controle do comportamento), a intenção para deixar de fumar pode ser baixa apesar das atitudes e normas subjetivas serem favoráveis.

Os objetivos do presente trabalho foram: (a) avaliar se as representações associadas ao tabaco funcionam como variáveis mediadoras na relação entre o ajustamento de casal e o suporte do parceiro; (b) avaliar o efeito mediador do suporte do parceiro na relação entre as representações do tabaco e as variáveis sócio-cognitivas em relação a deixar de fumar.

\section{Método}

\section{Participantes}

Neste estudo participaram 224 fumantes diários $(52,3 \%)$ e 169 ex-fumantes que tinham deixado de fumar há pelo menos 3 meses (47,3\%). Em relação aos fumantes, 52,7\% eram mulheres, $49,1 \%$ eram solteiros, $61,6 \%$ tinha o $12^{\circ}$ ano de escolaridade e $41,1 \%$ pertenceu ao intervalo de idades (21-30 anos). Vinte e um por cento dos fumantes começou a fumar aos 16 anos, $60,3 \%$ já tinham efetuado, pelo menos, 1 tentativa sem sucesso para deixar de fumar, $96 \%$ nunca frequentaram qualquer tratamento para deixar de fumar e $57,1 \%$ tinham um parceiro/a que não fumava.

Nos ex-fumantes, $68,6 \%$ eram homens, $78,2 \%$ eram casados, $39,1 \%$ tinha o $12^{\circ}$ ano de escolaridade e $27,2 \%$ pertenceu ao intervalo de idades (41-50 anos). 16\% dos ex-fumantes começaram a fumar aos 15 anos, 54,4\% efetuaram, pelo menos, 1 tentativa para deixar de fumar e 
$68 \%$ nunca frequentaram qualquer tratamento ou programa para deixar de fumar. Dos ex-fumantes $68 \%$ tinham um parceiro/a que não fumava.

\section{Procedimentos}

Os participantes foram recrutados num hospital central, numa empresa privada e numa universidade no Norte de Portugal. A participação foi voluntária e o preenchimento dos instrumentos efetuou-se num momento único. Os critérios de inclusão para a amostra foram: ser maior de 18 anos, ser fumante diário ou ser abstinente há pelo menos 3 meses. Sem conflito de interesse, a pesquisa foi aprovada pelo Comitê de Ética de cada local de coleta dos dados recebendo aprovação.

\section{Instrumentos}

Foram utilizados os seguintes instrumentos: Questionário Sociodemográfico (Pereira \& Afonso, 2009). Tinha como objetivo recolher informações ao nível de: estado civil, sexo, grau de instrução, tipo e quantidade de cigarros consumidos (fumante), duração do consumo/abstinência.

Brief Illness Perception Questionnaire (Brief-IPQ; Broadbent et al., 2006; versão portuguesa de Figueiras et al., 2010). Usado para avaliar as representações associadas ao tabaco (emocionais, cognitivas e a compreensão). É constituído por nove itens que se organizam em oito subescalas: Consequências, Duração, Controle Pessoal, Controle do Tratamento, Identidade, Preocupação, Compreensão, Resposta Emocional e Causas da doença. Utiliza uma escala (0-10), com exceção do item 9 (Causanão incluída neste estudo). As subescalas do instrumento são constituídas por um 1 item não permitindo efetuar estudos de fidelidade ou validade. O termo original "doença" foi substituído por "hábito de fumar" em fumantes e ex-fumantes para os efeitos do presente estudo. Um valor elevado em cada item representa maior intensidade no tipo de crença avaliada.

Partner Interaction Questionnaire (PIQ; Cohen \& Lichtenstein, 1990, versão de investigação de Pereira \& Afonso, 2006). Usado para avaliar apoio recebido para deixar de fumar. È composto por uma escala positiva ( 8 itens) e uma negativa (11 itens). Foi utilizada uma versão adaptada que permitisse o seu uso quer em fumantes quer em ex-fumantes. A escala positiva apresentou um alfa de 0,73 e a negativa um alfa de 0,84 . Um resultado elevado significa maior apoio, positivo ou negativo, por parte do parceiro.

Escala de Ajustamento Diádico-R (R-DAS; Busby, Christensen, Crane, \& Larson, 1995; versão de investigação de Pereira, 2003). Usado para avaliar o ajustamento de casal. É constituído por 14 itens subdivididos em 3 subescalas (satisfação, coesão e consenso). A cotação total varia entre 0 e 69 e maior o resultado das subescalas, maior o índice de ajustamento de casal. As subescalas apresentaram valores de alfa de 0,81 (satisfação), 0,79 (coesão) e 0,81 (consenso). Um resultado elevado, em cada subescala, significa maior satisfação, coesão e consenso do casal.

Questionário do Comportamento Planeado para Deixar de Fumar (QCP-DF; Ajzen, 2002; Pereira \& Afonso, versão de investigação, 2010). Este questionário foi construído para os objectivos do presente estudo para avaliar dimensões cognitivas associadas à intenção para deixar de fumar. Foi construído com base nos construtos da Teoria do Comportamento Planejado (TCP). A versão adaptada é constituída por 8 subescalas: Planejamento do Coping e Ação (alfa de 0,91); Crenças de Comportamento (alfa de 0,87); Atitudes face ao Comportamento (alfa de 0,91); Controle Comportamental Percebido (alfa de 0,81), Normas Subjetivas (alfa de 0,68); Crenças Normativas/Controle (alfa de 0,75) e Intenção. Um resultado elevado no questionário indica mais intensidade em cada subescala.

\section{Análise de Dados}

Para estudar o efeito mediador das representações do tabaco e do suporte do parceiro, realizou-se um conjunto de regressões segundo o método das etapas causais de Baron e Kenny (1986). Segundo estes autores, começa-se por testar o efeito direto da cada uma das variaveis independentes quando regredidas na variável dependente (passo 1). Se esta relação for signi- 
ficativa (via c), então a segunda e terceira equações de regressão são analisadas (passo 2). $\mathrm{Na}$ segunda equação, o mediador é regredido na VD (via a). A terceira equação implica regredir a VD simultaneamente na variável preditora (via c') e na variável mediadora (via b). As vias $a, b$, e c devem ser significativas. Por sua vez, a via c' deve ser zero (efeito mediador total) ou diminuir de forma significativa comparada com a via c (efeito mediador parcial). O teste de Sobel (1982) é calculado para verificar o efeito indireto da VI sobre a VD através da VM.

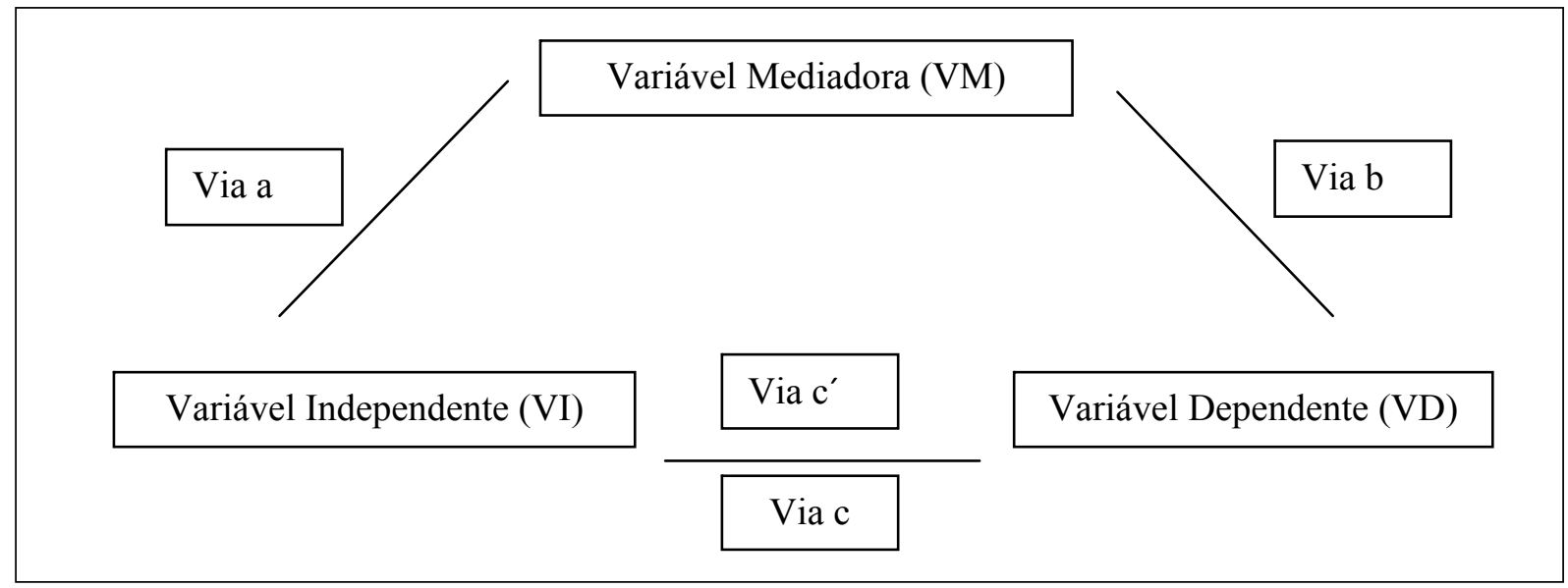

Figura1. Modelo de Mediação (Baron \& Kenney, 1986).

\section{Resultados}

\section{Efeitos da Mediação do Suporte do Parceiro na relação entre as Representações do Tabaco e as Variáveis Sócio-Cognitivas para Deixar de Fumar, em Fumantes}

O cálculo da regressão permitem verificar que o primeiro requisito para a mediação estava cumprido, dado que o valor estandardizado do coeficiente de correlação associado ao efeito das representações cognitivas com as variáveis sócio-cognitivas (via c) era significativo, em particular, com o controle comportamental percebido $(\beta=-0,269 ; p=0,000)$, atitudes $(\beta=0,159$; $\mathrm{p}=0,017)$, e planejamento do coping/ação $(\beta=$ $-0,163 ; p=0,014)$. Os coeficientes estandardizados associados à relação entre o preditor e o controle comportamental percebido (via a) também se mostraram significativos $(\beta=-0,189$; $p=0,005)$, para as atitudes $(\beta=-0,189 ; p=0,005)$ e para o planejamento do coping/ação $(\beta=-$ $0,189 ; p=0,005)$, estando assim cumprido o segundo requisito. Os valores dos coeficientes estandardizados associados com a relação entre o suporte positivo e as variáveis sócio-cogniti- vas (via b) se mostraram significativos para o controle comportamental percebido $(\beta=-0,270$; $p=0,000)$, para as atitudes $(\beta=0,227 ; p=0,001) \mathrm{e}$ para o planejamento do coping/ação $(\beta=0,030$; $p=0,652)$. Por fim, esta mesma equação de regressão mostrou que o valor dos coeficientes de correlação entre as representações cognitivas e as variáveis sócio-cognitivas (via c'; controlando o preditor proximal) é significativo para o controle comportamental percebido $(\beta=-0,320$; $p=0,000)$, para as atitudes $(\beta=0,202 ; p=0,002) \mathrm{e}$ para o planejamento do coping/ação $(\beta=-0,158$; $p=0,021)$. Assim, o suporte positivo do parceiro parece ter um efeito de mediação parcial da relação entre as representações cognitivas associadas ao tabaco e as variáveis sócio-cognitivas: controle comportamental percebido, atitudes e planejamento coping/ação (Teste de Sobel: $Z=$ $-1,66 ; p \leq 0,001)$. Neste sentido, podemos dizer que a relação entre as representações cognitivas e o controle comportamental percebido é mediada em $19 \%$ pelo suporte positivo do parceiro. A relação entre as representações cognitivas e as atitudes é mediada em $27 \%$ pelo suporte positivo do parceiro. Por sua vez, a relação entre as representações cognitivas e o planejamento do 
coping/ação é mediada em 3,5\% pelo suporte do parceiro.

Não se verificaram resultados significativos ao nível do efeito mediador do suporte positivo do parceiro na relação entre as representações emocionais, e compreensão e as variáveis sócio-cognitivas para deixar de fumar (normas subjetivas, crenças de comportamento, crenças normativas/controle e intenção). Por sua vez, em relação ao efeito mediador do suporte negativo do parceiro na relação entre as representações do tabaco (cognitivas, emocionais e compreensão) e as variáveis sócio-cognitivas para deixar de fumar (atitudes, normas subjetivas, crenças de comportamento, crenças normativas/controle, planejamento do coping/ação e intenção) também não se verificaram resultados significativos.

\section{Tabela 1}

Análise dos Efeitos da Mediação do Suporte Positivo do Parceiro na Relação entre as Representações Cognitivas do Tabaco e as Variáveis Sócio-Cognitivas (controle comportamental percebido) em Fumantes

\begin{tabular}{lcccc}
\multicolumn{1}{c}{ Passos } & $R^{2}$ & $\beta$ & $t$ & $p$ \\
\hline Primeiro passo (via c) & & & & \\
VD: controle comportamental percebido & 0,072 & $-0,269$ & $-4,166$ & 0,000 \\
$\begin{array}{l}\text { Preditor: representações cognitivas } \\
\text { Segundo passo (via a) }\end{array}$ & & & & \\
VD: suporte positivo & 0,036 & $-0,189$ & $-2,865$ & 0,005 \\
Preditor: representações cognitivas & & & & \\
Terceiro passo (via b e c') & & & & \\
VD: controle comportamental percebido & & & & \\
VM: suporte positivo (via b) & 0,143 & $-0,270$ & $-4,259$ & 0,000 \\
Preditor: representações cognitivas (via c') & & $-0,320$ & $-5,050$ & 0,000 \\
\hline
\end{tabular}

\section{Tabela 2}

Análise dos Efeitos da Mediação do Suporte Positivo do Parceiro na Relação entre as Representações Cognitivas do Tabaco e as Variáveis Sócio-Cognitivas (atitudes) em Fumantes

\begin{tabular}{lcccc}
\hline \multicolumn{1}{c}{ Passos } & $R^{2}$ & $\beta$ & $t$ & $p$ \\
\hline $\begin{array}{l}\text { Primeiro passo (via c) } \\
\text { VD: atitudes face ao comportamento }\end{array}$ & 0,021 & 0,159 & 2,399 & 0,017 \\
$\begin{array}{l}\text { Preditor: representações cognitivas } \\
\text { Segundo passo (via a) }\end{array}$ & & & & \\
VD: suporte positivo & 0,031 & $-0,189$ & $-2,865$ & 0,005 \\
Preditor: representações cognitivas & & & & \\
Terceiro passo (via b e c') & & & & \\
VD: atitudes face ao comportamento & & & & \\
VM: suporte positivo (via b) & 0,067 & 0,227 & 3,452 & 0,001 \\
Preditor: representações cognitivas (via c') & & 0,202 & 3,065 & 0,002 \\
\hline
\end{tabular}


Tabela 3

Análise dos Efeitos da Mediação do Suporte Positivo do Parceiro na Relação entre as Representações Cognitivas do Tabaco e as Variáveis Sócio-Cognitivas (planejamento do coping/ação) em Fumantes

\begin{tabular}{lcccc}
\hline Passos & $R^{2}$ & $\beta$ & $t$ & $p$ \\
\hline Primeiro passo (via c) & & & & \\
VD: Planejamento coping/ação & 0,022 & $-0,163$ & $-2,466$ & 0,014 \\
Preditor: representações cognitivas & & & & \\
Segundo passo (via a) & 0,031 & $-0,189$ & -2.865 & 0,005 \\
VD: suporte positivo & & & & \\
Preditor: representações cognitivas & & & & \\
Terceiro passo (via b e c') & & & & \\
VD: Planejamento coping/ação & 0,019 & 0,030 & 0,451 & 0,652 \\
VM: suporte positivo (via b) & & $-0,158$ & $-2,332$ & 0,021 \\
Preditor: representações cognitivas (via c') & & &
\end{tabular}

\section{Efeitos da Mediação}

das Representações do Tabaco na Relação entre o Ajustamento de Casal e o Suporte do Parceiro em Fumantes e Ex-fumantes

Fumantes. Para a variável mediadora representações emocionais do tabaco, os valores estandardizados dos coeficientes de correlação associados ao efeito do ajustamento de casal com o suporte positivo do parceiro (via c; $\beta=0,322 ; p=0,000$ ), entre o preditor e o possível mediador representações emocionais (via a; $\beta=-0,313 ; p=0,070)$ e, a relação entre as representações emocionais e o suporte positivo do parceiro (via $b$ ) não se mostraram significativos $(\beta=0,054 ; p=0,425)$. Em relação à variável mediadora, representações cognitivas do tabaco, os valores estandardizados dos coeficientes de correlação associados ao efeito do ajustamento de casal com o suporte positivo do parceiro (via c; $\beta=0,322 ; p=0,000)$, entre o preditor e as representações cognitivas (via a; $\beta=-0,044 ; p=0,512$ ) e, a relação entre as representações cognitivas e o suporte positivo do parceiro (via b) não se mostraram significativos $(\beta=-0,175 ; p=0,180)$. Em relação à variável mediadora compreensão, os valores estandardizados dos coeficientes de correlação associados ao efeito do ajustamento de casal com o suporte positivo do parceiro (via c; $\beta=0,322 ; p=0,000$ ), entre o preditor e a compreensão (via a; $\beta=-0,319 ; p=0,053$ ) e, a relação entre as compreensão e o suporte positivo do parceiro (via $b$ ) não se mostraram significativos $(\beta=0,049 ; p=0,462)$. Por fim, esta mesma equação de regressão mostrou que o valor dos coeficientes de correlação entre o ajustamento de casal e o suporte positivo do parceiro (via $\mathrm{c}^{\prime}$; controlando o preditor proximal: representações emocionais, representações cognitivas e compreensão) apresentou valores não significativos, respetivamente $(\beta=0,339 ; p=0,400 ; \beta$ $=0,315 ; p=0,070 ; \beta=0,338 ; p=0,058)$.

Em relação à variável dependente, suporte negativo do parceiro, os valores estandardizados dos coeficientes de correlação associados ao efeito do ajustamento de casal com o suporte negativo do parceiro (via $c ; \beta=0,164 ; p=0,017$ ), entre o preditor e o possível mediador (representações cognitivas; via a; $\beta=-0,044 ; p=0,512$ ) $\mathrm{e}$, a relação entre as representações cognitivas e o suporte negativo do parceiro (via $b$ ) não se mostraram significativos $(\beta=0,064 ; p=0,333)$. Os valores estandardizados dos coeficientes de correlação associados ao efeito do ajustamen- 
to de casal com o suporte negativo do parceiro (via $\mathrm{c} ; \beta=0,164 ; p=0,017$ ), entre o preditor e o possível mediador (representações emocionais; via a; $\beta=-313 ; p=0,000)$ e, a relação entre as representações emocionais e o suporte negativo do parceiro (via b) não se mostraram significativos $(\beta=0,164 ; p=0,170)$. Os valores estandardizados dos coeficientes de correlação associados ao efeito do ajustamento de casal com o suporte negativo do parceiro (via c; $\beta=0,164 ; p=0,017$ ), entre o preditor e o possível mediador (compreensão; via a; $\beta=-319 ; p=0,000)$ e, a relação entre a compreensão e o suporte negativo do parceiro (via b) não se mostraram significati$\operatorname{vos}(\beta=0,034 ; p=0,629)$. Por fim, esta mesma equação de regressão mostrou que o valor dos coeficientes de correlação entre o ajustamento de casal e o suporte negativo do parceiro (via $\mathrm{c}^{\prime}$; controlando o preditor proximal: representações cognitivas, emocionais e compreensão) apresentou valores não significativos, respetivamente $(\beta=0,167 ; p=0,012 ; \beta=0,196 ; p$ $=0,164 ; \beta=0,175 ; p=0,163)$.

Ex-fumantes. Em relação à variável mediadora representações cognitivas, os valores estandardizados dos coeficientes de correlação associados ao efeito do ajustamento de casal com o suporte positivo do parceiro (via c; $\beta=0,116$; $p=0,133$ ), entre o preditor e o possível mediador representações cognitivas (via a; $\beta=-0,003$; $p=0,965)$ e, a relação entre as representações cognitivas e o suporte positivo do parceiro (via b) não se mostraram significativos $(\beta=-0,120$; $p=0,121)$. Em relação à variável mediadora representações emocionais os valores estandardizados dos coeficientes de correlação associados ao efeito do ajustamento de casal com o suporte positivo do parceiro (via $c ; \beta=0,116 ; p=0,133$ ), entre o preditor e o possível mediador (representações emocionais; via a; $\beta=-0,180 ; p=0,019$ ) e, a relação entre as representações emocionais e o suporte positivo do parceiro (via b) não se mostraram significativos $(\beta=0,145 ; p=0,063)$. Em relação à variável mediadora compreensão os valores estandardizados dos coeficientes de correlação associados ao efeito do ajustamento de casal com o suporte positivo do parceiro (via c; $\beta=0,116 ; p=0,133)$, entre o preditor e o pos- sível mediador (compreensão; via a; $\beta=0,089$; $p=0,249)$ e, a relação entre a compreensão e o suporte positivo do parceiro (via $b$ ) não se mostraram significativos $(\beta=-0,093 ; p=0,230)$. Por fim, esta mesma equação de regressão mostrou que o valor dos coeficientes de correlação entre o ajustamento de casal e o suporte positivo do parceiro (via c'; controlando o preditor proximal: representações cognitivas, emocionais e compreensão) apresentou valores não significativos, respetivamente $(\beta=0,119 ; p=0,122$; $\beta=0,142 ; p=0,069 ; \beta=0,124 ; p=0,109)$. Em relação à variável dependente, suporte negativo do parceiro, os valores estandardizados dos coeficientes de correlação associados ao efeito do ajustamento de casal com o suporte negativo do parceiro (via c; $\beta=-0,045 ; p=0,557$ ), entre o preditor e o possível mediador (representações cognitivas; via a; $\beta=0,003 ; p=0,965)$ e, a relação entre as representações cognitivas e o suporte negativo do parceiro (via $b$ ) não se mostraram significativos $(\beta=0,095 ; p=0,224)$. Os valores estandardizados dos coeficientes de correlação associados ao efeito do ajustamento de casal com o suporte negativo do parceiro (via c; $\beta=-0,045$; $p=0,557$ ), entre o preditor e o possível mediador (representações emocionais; via a; $\beta=-180$; $p=0,019)$ e, a relação entre as representações emocionais e o suporte negativo do parceiro (via b) não se mostraram significativos $(\beta=0,058$; $p=0,459)$. Assim, as representações do tabaco (cognitivas, emocionais e compreensão) não tiveram um efeito mediador na relação entre o ajustamento de casal e o suporte do parceiro nos fumantes e nos ex-fumantes.

\section{Discussão}

Este estudo pretendeu estudar o efeito mediador das representações do tabaco e do suporte do parceiro.

No que diz respeito ao papel mediador do suporte do parceiro na relação entre as representações associadas ao tabaco (representações cognitivas) e as variáveis sócio-cognitivas (controle comportamental percebido, atitudes e planejamento do coping/ação), nos fumantes, verificou-se um efeito de mediação parcial. A 
relação negativa entre o suporte positivo do parceiro e as representações cognitivas associadas ao tabaco indica que existe mais suporte positivo do parceiro para deixar de fumar quando as representações cognitivas do tabaco são mais ameaçadoras. Estas últimas contemplam o fato de o fumante fumar há muito tempo, ter a perceção das consequências negativas do tabaco para a saúde, possuir pouco controle pessoal sobre fumar, percecionar o tratamento para deixar de fumar como pouco eficaz e quando não considera relevante a presença de sintomas físicos e psicológicos associados ao fumar. Estes dados podem sugerir que os fumantes não consideram que fumar é perigoso para a sua saúde. A presença de mais suporte positivo do parceiro indica menos controle comportamental percebido sugerindo que o fumante ao receber mais suporte do parceiro para deixar de fumar mesmo quando considerava que não tinha controle sobre o seu comportamento. Deixar de fumar não depende só da motivação que se tem para o fazer, mas também pelo fato de existir um controle real (Ajzen \& Madden, 1986). O controle comportamental percebido refere-se à facilidade, ou dificuldade, em levar a cabo o comportamento, neste caso, a deixar de fumar (Ajzen, 2002; Ajzen $\&$ Madden, 1986). A relação positiva entre o suporte positivo do parceiro e, o planejamento do coping/ação e atitudes face ao tabaco, referidos neste estudo, sugerem a importância do suporte do parceiro para deixar de fumar revelando que o apoio positivo é preditivo do sucesso da desabituação tabágica (Park et al., 2004; Rüge et al., 2008). Assim, de acordo com a literatura e, de acordo com os resultados encontrados neste estudo, podemos interpretar que existe mais suporte do parceiro para deixar de fumar quanto melhor é a avaliação do comportamento a executar (atitudes) e, ao mesmo tempo, existir um plano quanto ao modo de como deixar de fumar (planejamento; Sniehotta, Scholz, \& Schwarzer, 2005, 2006). A TCP refere quando se inicia a mudança de um comportamento, por exemplo deixar de fumar, os indivíduos poderão beneficiar de um plano que inclua estratégias de coping (coping planning) que ajudam o indivíduo na implementação das suas intenções (action planning; Van Osch, Lechner, Reubsaet, Wigger, \& de Vries, 2008). Estes dados podem sugerir que se o fumante quer deixar de fumar, se tiver apoio do parceiro, e se tiver um plano, o sucesso poderá ser maior.

No que respeita às correlações entre o ajustamento de casal e o suporte do parceiro para deixar de fumar, nos fumantes, os resultados vão ao encontro da literatura indicando que quanto mais suporte o fumante recebe para deixar de fumar por parte do parceiro, mais ajustamento se verifica ao nível do casal (Rohrbaugh et al., 2009; Rohrbaugh et al., 2001). O suporte do parceiro se mostrou uma variável importante na cessação do tabagismo (Park et al., 2004; Rüge et al., 2008) podendo influenciar o fumante a deixar de fumar, e a manter-se sem fumar (Homish \& Kenneth, 2005; Rüge et al., 2008). O suporte do parceiro também se revela como uma variável mediadora do sucesso da cessação do tabagismo (Shoham et al., 2006). Nos fumantes, tal como nos ex-fumantes, a relação negativa entre o ajustamento de casal e as representações emocionais do tabaco indicaram que quanto menos se sentiam afetados emocionalmente por fumar, e mais preocupação demostravam com o tabaco, i.e., quanto menos consideravam que o tabaco era ameaçador para a sua saúde, mais ajustamento ao nível do casal se verificava. No presente estudo o tabaco pode ser considerado um elemento de aproximação entre o casal na medida em que um dos membros do casal pode querer ajudar o fumante a deixar de fumar. Estes dados vão ao encontro da literatura que referem que o fumante poder ser ajudado pelo parceiro a deixar de fumar (Wenner et al., 2004). No presente estudo, os ex-fumantes demostraram mais compreensão associada ao tabaco e mais suporte negativo do parceiro para deixar de fumar. Assim, embora os ex-fumantes (quando fumavam) demostrassem que compreendiam porque fumavam, o parceiro proporcionou um apoio mais crítico podendo ser desmotivador (Copotelli \& Orleans, 1985; Roski et al., 1996). Estes dados sugerem que o fumante que receba mais suporte negativo do parceiro pode apresentar mais dificuldades em iniciar a cessação tabágica. 


\section{Considerações Finais}

O fato de a amostra ser de conveniência, recrutada apenas na zona Norte de Portugal limita a generalização dos resultados. A aplicação de medidas de autorrelato do tipo retrospetivo a que os ex-fumantes responderam pode ser considerada uma limitação do presente estudo.

Investigações futuras devem contemplar amostras diversificadas em que o suporte do parceiro é também avaliado pelo próprio parceiro. Além disso, seria importante incluir as representações do parceiro em relação ao tabaco, no sentido de analisar de que forma a congruência no casal, a este nível, pode ou não prever a cessação tabágica.

Os resultados apontam para a importância do efeito mediador do suporte do parceiro junto dos fumantes que querem deixar de fumar indo ao encontro da literatura (Afonso, 2008; Mermelstein et al., 1983; Park et al., 2004; Rohrbaugh et al., 2001; Rüge et al., 2008). Desta forma, e de acordo com os resultados encontrados, o apoio do parceiro é um fator importante junto dos fumantes com intenção para deixar de fumar, revelando-se como um dos melhores preditores para a abstinência tabágica (Lawhon et al., 2009; Nollen et al., 2005).

\section{Referências}

Afonso, M. F. (2008). Variáveis individuais e familiares no comportamento tabágico (Dissertação de mestrado, Universidade do Minho, Braga, Portugal).

Ajzen, I. (1991). The theory of planned behavior. Organizational Behavior and Human Decision Processes, 50, 179-211.

Ajzen, I. (2002). Constructing a TpB Questionnaire: Conceptual and methodological considerations. Retrieved from http://www.apcc.online.pt/uplo$\mathrm{ad} / \mathrm{membro.id/ficheiros/i005562.pdf}$

Ajzen, I., \& Madden, T. J. (1986). Prediction of goaldirected behavior: Attitudes, intentions, and perceived behavioral control. Journal of Experimental Social Psychology, 2, 453-474.
Baron, M., \& Kenney, D. A. (1986). The moderatormediator variable distinction in social psychological research: Conceptual, strategic, and statistical considerations. Journal of Personality and Social Psychology, 51, 1173-1182.

Becoña, E. (2003). El tratamiento psicológico de la adicción a la niciotina. Papeles del Psicologo, $85,48-69$.

Broadbent, E., Petrie, K., Main, J., \& Weinman, J. (2006). The brief illness perception questionnaire. Journal of Psychosomatic Research, 60, 631-637.

Busby, D., Christensen, C., Crane, D., \& Larson, J. (1995). A revision of the Dyadic Adjustment Scale for use with distressed and nondistressed couple: Construct hierarchy and multidimensional scales. Journal of Marital and Family Therapy, 21(3), 289-308.

Cohen, S., \& Lichtenstein, E. (1990). Partner behaviours that support quitting smoking. Journal of Consulting and Clinical Psychology, 58(3), 304309.

Copotelli, H., \& Orleans, C. (1985). Partner support and other determinants of smoking cessation maintenance among women. Journal of Consulting and Clinical Psychology, 53(4), 455-460.

Direção Geral de Saúde. (2009). Elementos estatísticos. Informação geral de saúde/2007. Lisboa, Portugal: Autor. Recuperado em http://www. dgs.pt/

Figueiras, M., Marcelino, D. S., Claudino, A., Cortes, M. A., Maroco, J., \& Weinman, J. (2010). Patients' illness schemata of hypertension: The role of beliefs for the choice of treatment. Psychology \& Health, 25(4), 507-517. doi: 10.1080/08870440802578961(IF: 1.591)

Fila, S. A., \& Smith, C. (2006). Applying the theory of planned behavior to healthy eating behaviors in urban Native American youth. International Journal of Behavioural Nutrition and Physical Activity, 3(11), 1-10.

Fishbein, M., \& Ajzen, I. (1975). Belief, attitude, intention, and behavior: An introduction to theory and research. Reading, MA: Addison-Wesley.

Hill, A. J., Boudreau, F., Amyot, E., De'ry, D., \& Godin, G. (1997). Predicting the stages of smoking acquisition according to the theory of planned behavior. Journal of Adolescent Health, 21, 107-115. 
Homish, G., \& Kenneth, L. (2005). Spousal influence on smoking behaviours in a US community sample of newly married couples. Social Science and Medicine, 61, 2557-567.

Instituto Nacional de Cardiologia Preventiva. (2002). A situação em Portugal em relação com outros países europeus. Lisboa, Portugal: Autor.

Lawhon, D., Humfleet, G., Hall, S., Reus, V., \&, Muñoz, R. (2009). Longitudinal analysis of abstinence-specific social support and smoking cessation. Health Psychology, 28(4), 465-472. doi: 10.1037/a0015206

Lopez, A. D., Mathers, C. D., Ezzati, M., Jamison, D. T., \& Murray, C. J. (2006). Global and regional burden of disease and risk factors, 2001: Systematic analysis of population health data. Lancet, $367,1747-1757$.

Martinet, Y., \& Bohadana, A. (2003). O tabagismo. Da prevenção à abstinência. Lisboa, Portugal: Climepsi.

Meneses, R. F., Ribeiro, J. P., \& Silva, A. M. (2002). Revisão da literatura sobre avaliação da qualidade de vida (QDV) de adultos com epilepsia. II Facilidades na abordagem do tema. Psicologia, Saúde \& Doença, 3(2), 119-139.

Mermelstein, R., Lichtenstein, E., \& McIntyre, K. (1983). Partner support and relapse in smoking cessation programs. Journal of Consulting and Clinical Psychology, 51, 465-466.

Moan, I. S., \& Rise, J. (2005). Quitting smoking: Applying an extended version of the theory of planned behaviour in predicting intention and behaviour. Journal of Applied Biobehavioral Research, 10, 39-68.

Nollen, N., Catley, D., Davies, G., Matthew, H., Jasjit, G., \& Ahluwalia, S. (2005). Religiosity, social support, and smoking cessation among urban African American smokers. Addicted Behaviors, 30, 1225-1229.

Park, E., Tudiver, F., Schultz, J., \& Campbell, T. (2004). Does enhancing partner support and interaction improve smoking cessation? A metaAnalysis. Annals of Family Medicine, 2(2), 170174.

Pereira, M. G. (2003). Revised Dyadic Adjustment Scale. Versão de Investigação. Braga, Portugal: Universidade do Minho.
Pereira, M. G., \& Afonso, F. (2006). Partner Interaction Questionnaire. Versão de Investigação. Braga, Portugal: Universidade do Minho.

Pereira, M. G., \& Afonso, F. (2009). Questionário Sociodemográfico. Versão de Investigação. Braga, Portugal: Universidade do Minho.

Pereira, M. G., \& Afonso, F. (2012). Questionário do Comportamento Planeado para Deixar de Fumar (QCP-DF). Versão de Investigação. Braga, Portugal: Universidade do Minho.

Rise, J., Kovac, V., Kraft, P., \& Moan, I. S. (2008). Predicting the intention to quit smoking and quitting behaviour: Extending the theory of planned behaviour. British Journal of Health Psychology, 13, 291-310. doi: 10.1348/135910707X187245

Rohrbaugh, M., Shoham, V., \& Dempsey, C. (2009). Gender differences in quit support by partners of health-compromised smokers. Journal of Drug Issues, 39(2), 329-346.

Rohrbaugh, M., Shoham, V., Trost, S., Muramoto, M., Cate, R., \& Leischow, S., (2001). Couple dynamics of change-resistant smoking: Toward a family consultation model. Family Process, 40(1), 15-31.

Roski, J., Schmid, L. A., \& Landom, H. A. (1996). Long-term associations of helpful and harmful spousal behaviors with smoking cessation. $A d-$ dicted Behaviors, 21, 173-185.

Rüge, J., Ulbricht, S., Schummann, A., Rumpf, H. J., John, U., \& Meyer, C. (2008). Intention to quit smoking: Is the partners smoking status associated with the smoker's intention to quit? International Journal of Behavioral Medicine, 15, 328-325.

Shoham, V., Rohrbaugh, M. J., Trostm S. E., \& Muramoto, M. (2006). A family consultation intervention for health-compromised smokers. Journal of Substance Abuse Treatment, 31, 395-402.

Sniehotta, F. F., Scholz, U., \& Schwarzer, R. (2005). Bridging the intention-behaviour gap: Planning, self-efficacy, and action control in the adoption and maintenance of physical exercise. Psychology \& Health, 20, 143-160.

Sniehotta, F. F., Scholz, U., \& Schwarzer, R. (2006). Action plans and coping plans for physical exercise: A longitudinal intervention study in cardiac-rehabilitation. British Journal of Health Psychology, 11, 23-37. 
Sobel, M. E. (1982). Asymptotic confidence intervals for indirect effects in structural equations models. In S. Leinhart (Ed.), Sociological methodology 1982 (pp. 290-312). San Francisco, CA: Jossey-Bass.

Thomas, J., Patten, C. A., Mahnken, J. D., Offord, K. P., Hou, A., Lynam, I. M., ...Croghan, I. T. (2009).Validation of the support provided measure among spouses of smokers receiving a clinical smoking cessation intervention. Psychology Health and Medicine, 14(4), 443-453.

Trigo, M. (2005). O que provoca realmente a mudança nos fumadores? Algumas reflexões. Revista Portuguesa de Clínica Geral, 21, 161-182.

Van Osch, L., Lechner, L., Reubsaet, A., Wigger, S., \& de Vries, H. (2008). Relapse prevention in a national smoking cessation contest: Effects of coping planning. British Journal of Health Psychology, 13, 525-535.
Wenner, C., Rohrbaugh, M. J., Shoham, V., \& Trost, A. (2004). Emotion regulation by dual- and single-smoker couples in a laboratory smoking task. Phoenix, AZ: Western Psychological Association.
Recebido: 30/10/2012

$1^{a}$ revisão: 21/12/2012 Aceite final: 11/01/2013 